\title{
Formation et initiation à la tâche : éléments de ritualisation favorisant le développement d'une culture de santé et sécurité au travail auprès des travailleurs immigrants
}

Training and initiation to the task: ritual elements promoting the development of a culture of health and safety at work for immigrant workers Formación e iniciación a la tarea : elementos de ritualización que favorecen el desarrollo de una cultura de salud y seguridad en los trabajadores inmigrantes

Sylvie Gravel, Jacques Rhéaume et Gabrielle Legendre

\section{OpenEdition \\ Journals}

Édition électronique

URL : http://journals.openedition.org/pistes/1821

DOI : $10.4000 /$ pistes. 1821

ISSN : 1481-9384

Éditeur

Les Amis de PISTES

Édition imprimée

Date de publication : 1 novembre 2011

Référence électronique

Sylvie Gravel, Jacques Rhéaume et Gabrielle Legendre, « Formation et initiation à la tâche : éléments de ritualisation favorisant le développement d'une culture de santé et sécurité au travail auprès des travailleurs immigrants », Perspectives interdisciplinaires sur le travail et la santé [En ligne], 13-2 | 2011, mis en ligne le 01 novembre 2011, consulté le 19 avril 2019. URL : http://journals.openedition.org/ pistes/1821; DOl : 10.4000/pistes.1821

Ce document a été généré automatiquement le 19 avril 2019. d'Utilisation Commerciale - Pas de Modification 4.0 International. 


\section{Formation et initiation à la tâche : éléments de ritualisation favorisant le développement d'une culture de santé et sécurité au travail auprès des travailleurs immigrants}

Training and initiation to the task: ritual elements promoting the development of a culture of health and safety at work for immigrant workers Formación e iniciación a la tarea : elementos de ritualización que favorecen el desarrollo de una cultura de salud y seguridad en los trabajadores inmigrantes

Sylvie Gravel, Jacques Rhéaume et Gabrielle Legendre

\section{Introduction}

Depuis des années, les professionnels de la santé au travail s'inquiètent des limites des petites entreprises (PE) pour implanter et gérer des mesures de SST. Cette inquiétude est d'autant plus justifiée que les PE constituent, dans bien des pays, le principal employeur ${ }^{1}$. De plus, dans les grands centres urbains d'Europe et d'Amérique du Nord, les PE embauchent un nombre important de travailleurs immigrants. Or, ces travailleurs issus des communautés ethnoculturelles ou de l'immigration semblent ignorer le rôle qu'ils peuvent jouer et la participation qui peut être la leur dans l'implantation des mesures de SST (Soto Mas et al., 1996; Stange et al., 1991). Des travaux démontrent qu'au sein de ces $\mathrm{PE}$, plusieurs facteurs internes et externes contribuent à freiner l'implantation des mesures correctrices et préventives de santé au travail (Walters, 2001), mais rarement ces travaux prennent en considération l'impact de la méconnaissance des droits et des obligations des travailleurs immigrants dans l'émergence d'une culture de SST. 
2 Trois facteurs internes sont énumérés par Charpentier et coll. (Charpertier-Roy et al., 2001) comme des freins importants à l'implantation des mesures de SST dans les PE. Le premier est l'interprétation des dirigeants des entreprises quant à leurs obligations et leurs responsabilités à prendre en charge les processus d'analyse des problèmes et d'implantation de solutions. Le deuxième frein est la perception des travailleurs de leurs devoirs de participation dans ces processus. Et finalement, les moyens structurels dont dispose l'entreprise, lui permettant de respecter ses obligations et ses responsabilités en matière de SST (Charpertier-Roy et al., 2001), constituent le troisième frein.

3 Cet article tentera de montrer que les compétences des dirigeants en matière de SST et leur formation acquise dans leur pays d'origine orientent l'adoption de rituels de prévention dès l'embauche des nouveaux travailleurs. Cet élément lié au contexte d'immigration se rajoute à l'influence des trois facteurs freinant l'implantation des mesures de SST dans les PE. Les résultats d'une étude menée auprès de 28 petites entreprises indiquent que les employeurs consacrent peu d'efforts à l'initiation à la tâche des nouveaux employés immigrants et à leur formation en cours d'emploi, deux moments phares pour affirmer une culture de SST au sein d'entreprises multiethniques.

\section{Problématique}

4 La perception des dirigeants quant à leurs obligations et leurs responsabilités se limite très souvent à fournir des équipements de protection individuelle (ÉPI), d'en surveiller l'usage quotidien, d'entretenir et de remplacer les équipements et les outils dangereux (Charpertier-Roy et al., 2001, Eakin, 1992, Eakin et al., 2000). Les mesures de prévention et de protection sont perçues par les dirigeants des PE comme une dépense et non comme un investissement (Flynn, 2007; Smith, 2007). La perception de la non-rentabilité des investissements en SST est d'autant plus forte lorsqu'il s'agit d'une main-d'œuvre facilement remplaçable comme les immigrants (Robinson, 1986). La participation des travailleurs à l'implantation des mesures de SST est fortement conditionnée par la présence d'un syndicat ou d'une association accréditée de travailleurs et d'un comité de santé et de sécurité au travail, une présence rarissime dans les PE (Stange et al., 1991). Aux facteurs internes s'ajoutent les ressources humaines et les moyens mis en place par les entreprises pour gérer les mesures de SST. Parmi les moyens jugés les plus efficaces pour créer cette dynamique propice à la prévention de la SST, il y a la présence d'un responsable de la SST formellement désigné, d'un comité de SST opérationnel, et l'implantation d'activités structurantes ponctuelles (enquêtes), de routine (inspections, vérification) ou de surveillance des mesures de SST (registres, audits). Ces études sur les dynamiques d'implantation des mesures de SST ont été réalisées sans égard à l'origine ethnique et linguistique des travailleurs, sans connaître le degré de maîtrise qu'ils ont de la culture de SST de la société d'accueil. Cela est d'autant plus préoccupant que les PE embauchent une proportion importante de travailleurs immigrants et que ceux-ci sont surexposés aux risques de lésions professionnelles (Flynn, 2007; Smith, 2007) et que ces lésions sont généralement plus graves, voire irréversibles (Windau, 1997).

5 En ce qui concerne les dynamiques d'implantation et de gestion des mesures de SST dans les PE, deux facteurs externes sont importants : a) la concurrence des marchés et b) les pressions légales dictées par les lois de SST (inspections, sanctions financières, arrêts de production, etc.) (Baril-Gingras et al., 2006). Ces pressions font en sorte que les PE se consacrent à développer des stratégies de survie au détriment de leurs obligations en 
matière de SST (Quinlan et Mayhew, 1999; Quinlan, 2004) dont celles d'harmoniser les normes et des pratiques de SST (Walters, 2004; Walters, 2008). La circulation internationale des personnes et des équipements complexifie cette harmonisation des normes de SST. Ces problèmes d'harmonisation sont davantage liés à la perception culturelle des risques qu'à des pressions légales exercées par les instances de SST (Walters, 1996).

6 Les écarts culturels de perception des risques sont connus dans divers domaines de la santé et particulièrement dans le domaine de la promotion des saines habitudes de vie. En SST, les connaissances et les capacités du propriétaire-dirigeant à évaluer les risques de SST, à partager la responsabilité d'appliquer des mesures correctrices, à lancer des projets et à entendre les revendications des travailleurs sont autant de dimensions culturelles en prévention de la SST. Les superviseurs sont considérés comme les meilleurs promoteurs des réflexes de SST, de gestes quotidiens de correction et de prévention dans les PE (Smtih, 2007). Malgré les nombreux travaux en management de la SST, rarement l'immigration est abordée comme un élément de différenciation de la perception des risques, un marqueur de la culture de SST chez les travailleurs, les superviseurs et les dirigeants nés et formés hors Canada (Vickers et al., 2003). Il existe pourtant bien des travaux sur la prévalence élevée des lésions professionnelles chez les travailleurs immigrants (Flynn, 2007; Smith, 2007; Windau, 1997) et des problèmes d'accès à l'indemnisation (Gravel, 2006; Lashay et al., 2002; Sum, 1996). Pourtant, très peu ont documenté la perception du risque chez les travailleurs immigrants, sauf chez les hispanophones et les Afro-Américains des États-Unis (Eggerth, 2007; Brown et al., 2002). Néanmoins, on peut facilement supposer que les trajectoires migratoires et les expositions antérieures aux risques modifieront les perceptions des travailleurs immigrants, leur propension à respecter les règles de SST et à prendre des initiatives.

7 Dans les travaux et les lois de SST, les comités paritaires et les formations sont considérés comme des leviers importants de communication et de transmission d'une culture de SST (Simard et Marchand, 1997). Toutefois, les PE qui implantent des comités paritaires le font souvent en fonction des obligations légales liées à leur secteur de production ou parce qu'elles sont syndiquées. Celles qui le font par conviction ont généralement une culture de SST très forte (Desmarais et Foucault, 2009). Mais là encore, très peu de travaux se sont intéressés à la composition et aux dynamiques des comités de SST dans les entreprises embauchant des travailleurs immigrants (Legendre, 2001). Selon les résultats de Legendre (2011), les comités de SST comptent rarement des membres issus de l'immigration, sauf s'ils sont présents à titre d'employeurs.

8 Les formations en SST offertes aux travailleurs sont souvent une occasion de participer et de faire valoir leur point de vue et leurs besoins auprès de leurs superviseurs hiérarchiques (Walters, 2004; Eakin et MacEachen, 1998). Encore faut-il que les travailleurs puissent communiquer aisément, ce qui n'est pas toujours le cas avec les travailleurs immigrants (Nash, 1996). Maints travaux ont démontré, dont ceux de Chatigny et coll. (2006), que la formation des employés contribue de façon significative au développement d'une culture de SST, peu importe l'approche: formation certifiée formelle, apprentis ou compagnonnage (Chatigny et al., 2006). La clé du succès est l'intégration des apprentissages aux tâches quotidiennes des travailleurs (Vézina et al., 2000, Pâques et al., 2005). La formation incite les travailleurs, même ceux non syndiqués, à participer aux changements en matière de SST dans les entreprises qui les embauchent (Walters, 1992). Là encore, ces travaux ne documentent pas les méthodes d'apprentissage 
dans des milieux multiethniques parce que ceux-ci participent rarement, ne se sentant pas concernés (Soto Mas et al., 1996; Stange et al., 1991; Williams et al., 2001).

9 Force est de constater que les dynamiques entre les employeurs, les superviseurs et les employés lors de l'implantation et dans la gestion des mesures de SST dans les PE embauchant dans une proportion importante des travailleurs immigrants, sont peu documentées. La culture de la SST en contexte interculturel au sein de PE est ignorée. Cela est d'autant plus préoccupant que la croissance économique de la société québécoise et nord-américaine comme maints pays européens repose en bonne partie sur la présence constante et pas juste saisonnière d'une main-d'œuvre étrangère (Taylor, 1996).

\section{Question de recherche et cadre d'analyse}

Dans le projet de recherche, nous avons postulé que les trajectoires migratoires ${ }^{2}$ (le pays de naissance et de formation professionnelle, la durée de séjour au Canada, la langue maternelle et de travail) façonnent la perception des dirigeants, des superviseurs et des travailleurs en matière de SST et la dynamique de prise en charge dans les PE embauchant majoritairement des immigrants. La question de recherche que nous traitons dans le présent article :

Quelle est l'importance accordée à la formation et l'initiation à l'embauche considérées comme des moments structurants et ritualisés d'une culture de prévention SST?

11 L'analyse des interactions entre les acteurs est au cœur de tout le projet de recherche. Pour ce volet traitant de la formation et de l'initiation à l'embauche, nous avons jugé opportun d'en faire l'analyse en nous inspirant des notions de l'ethnologie des rites et rituels initiatiques. Appliquer l'analyse des rites et des rituels au management de la SST est une approche audacieuse, mais peut être une contribution originale à l'étude de la culture de la SST dans les PE.

L'analyse des rites et des rituels de passage dans la culture ${ }^{3}$ est généralement centrée sur les événements significatifs de la vie, comme la naissance, le mariage, la mort et le passage à l'âge adulte, dans les sociétés traditionnelles (Van Gennep, 1924; Turner, 1990). Nos sociétés modernes ont-elles aussi encore besoin des rites et des rituels pour marquer les grands moments (Segalen, 2005) parce qu'ils

" [...] sont un ensemble de conduites individuelles et collectives relativement codifiées qui, à l'aide d'un support corporel (verbal, gestuel, posture), a un caractère répétitif, et est porteur d'une forte charge symbolique à la fois pour les acteurs et les témoins » (p. 21).

13 On retrouve ici tous les ingrédients efficaces pour inculquer un savoir-faire et un savoirêtre en matière de SST: des conduites individuelles et collectives dictées et non raisonnées, appuyées par un ensemble de dispositifs matériels (ÉPI, dispositif d'arrêt, etc.) et de communication (information, comité de SST), que l'on renouvelle par période ou selon certaines occasions symboliques. L'analyse des résultats de recherche montre que la formation et l'initiation à la tâche dans les PE embauchant majoritairement des travailleurs immigrants constituent un indicateur majeur de l'établissement d'une culture ritualisée en SST.

14 La motivation des hauts dirigeants est un élément central dans le développement des compétences et des capacités en SST, mais elle est suffisante pour créer cette culture de la 
SST. Ce qui semble faire défaut, c'est la capacité des entreprises à rendre " sacrée » la santé des travailleurs.

« Les rites sont des règles de conduite qui prescrivent comment l'homme doit se

comporter avec les choses sacrées » (Segalen, 2005, p. 10).

Néanmoins, les entreprises disposent d'un certain nombre d'éléments contribuant aux rituels : des personnes désignées (préventionnistes et formateurs), des objets ritualisés (équipements de protection individuelle, outils, et registres d'incidents et d'accidents), des rites d'initiation (formation à l'embauche, formation en cours d'emploi, supervision et compagnonnage) et des rites de sanction (inspection et cadenassage).

« Le rite comprend un certain nombre d'opérations, de gestes, de mots et d'objets convenus pour qu'il y ait croyance à une sorte de transcendance » (Segalen, 2005, p. 21).

16 Cette transcendance dans une culture de la SST est la prévention. Elle s'inscrit dans les réflexes quotidiens sans être nécessairement attachée à un mode de gestion basé sur la diligence raisonnable.

"L'essentiel du rite est que les individus soient réunis, expriment des actes communs et qu'ils se les réaffirment périodiquement » (Segalen, 2005, p.14).

L'aspect répétitif du rituel est probablement ce qui fait le plus souvent défaut dans les $\mathrm{PE}$, la capacité à renouveler les valeurs, les gestes et les actions préventives en SST.

Pour Fellous (2001), il n'y a pas de doute, notre société est en quête de nouveaux rituels sans églises, elle « [...] le fait à chaque fois qu'il y a un nouvel enjeu sociétal » (p. 31). Dans nos entreprises contemporaines, le nouvel enjeu est le renouvellement et le vieillissement de la main-d'œuvre. Cet enjeu à lui seul amène les entreprises à revoir leurs stratégies pour maintenir leurs travailleurs en emploi le plus longtemps possible, et ce, sans absences ni lésions professionnelles (Cloutier, 2009). La formation à l'embauche doit être cohérente avec les apprentissages de SST afin de veiller à un apprentissage rapide, efficace et sans douleur pour garder les nouveaux candidats dans des emplois peu attractifs (Chatigny et al., 2006). Les rituels de SST en entreprise et leur transcendance à la prévention sont souvent à l'ordre du jour des entreprises qui déjà doivent relever ce défi du vieillissement de la main-d'œuvre et de son remplacement.

Notre étude ne s'appuie pas sur des observations continues sur de longues périodes des pratiques ritualisées en milieu « naturel ». Nous avions un accès limité aux entreprises et aux acteurs concernés par cette dynamique de la prise en charge des mesures de SST dans les PE qui embauchent majoritairement des travailleurs immigrants. Nous avons interrogé les personnes responsables de la SST au sein de leur entreprise sur les moyens dont elles disposent pour former et initier les travailleurs. Nous n'avons pas eu accès ni aux formateurs, ni aux apprenants, ni pu observer une séance d'initiation à la tâche ou de formation. Nous transposons les observations des répondants de SST au cadre d'analyse des rites et des rituels, avec tous les biais d'observation et de sélection que cela suppose.

\section{Méthode de recherche}

Notre recherche porte sur les PE embauchant de façon significative des travailleurs immigrants. Il s'agit d'une étude prospective avec un échantillon raisonné de 28 entreprises divisées en deux groupes: d'observation et de comparaison. Les PE de l'échantillon sont du secteur privé, syndiquées ou non, embauchant moins de 50 travailleurs. Les PE représentant le groupe à l'étude $(\mathrm{n}=19)$ ont une main-d'œuvre 
composée d'au moins un quart de travailleurs immigrants, c'est-à-dire de travailleurs nés hors du Canada. À l'inverse, les PE du groupe de comparaison $(\mathrm{n}=9)$ ont une main-d'œuvre composée majoritairement de travailleurs nés au Canada $(\geq 75 \%)$. Le critère de travailleur immigrant est sans égard au statut (citoyen, immigrant reçu, réfugié ou en attente de statut) et sans égard à la durée de séjour au Canada.

Les critères d'inclusion des PE sont: 1) être situées dans l'un des deux territoires industriels de la région métropolitaine de Montréal couverts par les centres de santé et de services sociaux (CSSS) de la Montagne et de l'Ouest-de-l'île; 2) avoir bénéficié d'une intervention de l'équipe de professionnels de la santé et la sécurité au travail de l'un ou l'autre des CSSS entre novembre 2008 et juillet 2010. Les entreprises participantes $(n=28)$ représentent environ $10 \%$ des entreprises des deux territoires qui ont moins de 50 travailleurs et qui font partie des groupes prioritaires ciblés par la LSST sur les comités de santé et sécurité au travail.

22 Toutes les entreprises répondant aux critères d'inclusion ont été sollicitées. La sollicitation s'est faite par l'intermédiaire des professionnels de SST des deux CSSS. La grille d'entrevue était préalablement acheminée par courriel aux répondants de SST afin de les rassurer et de les guider sur les informations recherchées.

23 La répartition des entreprises dans le groupe à l'étude et le groupe de comparaison s'est faite a posteriori car l'origine des travailleurs n'est pas une information consignée par les entreprises et par les instances administratives de la SST (CSSS, CSST). Les répondants ont pour la plupart utilisé le registre interne des employés pour établir, pour nos besoins, le nombre de travailleurs nés à l'étranger. Il ne s'agit pas d'une information validée avec des documents de l'état civil, mais d'une connaissance fine des employés puisque ces répondants les côtoient quotidiennement depuis de nombreuses années. Plusieurs répondants ont validé, préalablement à l'entretien, les pays de naissance, les langues d'usage entre les employés et entre les superviseurs, surtout pour les équipes de nuit, qu'ils côtoient moins souvent. Tous les entretiens se sont déroulés en français ou en anglais. Les quelques entreprises où le propriétaire-dirigeant s'exprimait peu ou pas dans l'une ou l'autre des langues officielles ont refusé de participer avant même que nous puissions proposer de nous adjoindre un interprète pour mener l'entretien. Ça a été notamment le cas avec une entreprise où le propriétaire et tout le personnel étaient d'origine afghane. Nous avons certes ici un biais de sélection, car nous n'avons pas réussi à inclure dans notre échantillon les entreprises allophones. Toutefois, nous sommes sûrs que cette situation était exceptionnelle, généralement au moins un des dirigeants ou superviseurs pouvait s'exprimer aisément dans l'une ou l'autre des deux langues officielles.

24 Deux sources de données sont ici exploitées, les entretiens en face à face avec les répondants de la SST dans les PE et les entretiens avec les professionnels de la SST des CSSS qui œuvrent dans les PE de notre échantillon. Ces deux sources de données ont été constituées à partir d'entrevues semi-dirigées d'une heure, menées à l'aide d'une grille structurée d'observation systématique, par des chercheurs accompagnés d'un assistant de recherche. Les entretiens menés auprès des répondants de la SST des entreprises et les professionnels de SST des CSSS ont été réalisés sur les lieux de travail.

À l'origine, les entretiens auprès des répondants de la SST des PE devaient être menés par les professionnels de SST des CSSS partenaires, ceux qui interviennent dans ces usines dans le cadre de leur fonction. Les prétests ont rapidement révélé un problème de 
faisabilité. La sollicitation et la réalisation des entretiens s'est avérée trop lourde, trop prenante pour confier cette tâche aux professionnels de SST. L'équipe de recherche a rapidement repris en charge la sollicitation et la réalisation des entretiens. Ce changement de rôle entre les professionnels et l'équipe de recherche a eu des effets bénéfiques. Les répondants de la SST des PE se sont sentis dégagés envers les autorités de santé, l'équipe de recherche ne pouvant pas être une autorité normative ou suggestive pour implanter ou évaluer des mesures SST.

Plusieurs thèmes sont abordés dans l'étude dont la présence et la composition d'un comité de SST, la formation et l'information des employeurs, superviseurs et travailleurs, les arguments favorables et défavorables à la SST, les compétences et les moyens dont disposent les responsables de la SST. Les données traitées dans le présent article concernent davantage l'initiation des nouveaux employés aux postes de travail, la formation en SST qui leur est dispensée, les thèmes et les méthodes de formation qui sont proposés aux travailleurs. Les données ont été traitées sur les logiciels Excel et Nvivo8 à partir de ces thèmes.

\section{Résultats}

À l'origine, la sollicitation des entreprises à l'étude se faisait sur le territoire du CSSS de la Montagne réputé pour avoir une très grande concentration de travailleurs immigrants. Les entreprises du groupe de comparaison étaient recrutées sur le territoire du CSSS de l'Ouest-de-l'̂̂le. Or, il s'est avéré que le recrutement des entreprises avec peu de travailleurs immigrants a été laborieux, et ce, sur les deux territoires. Malgré cette difficulté nous avons atteint notre objectif: 19 entreprises sur 28 (68\%) de notre échantillon ont une main-d'œuvre composée de plus de $25 \%$ de travailleurs immigrants. Le groupe à l'étude comprend les entreprises qui embauchent des immigrants ( $>25 \%)$, dont l'une sur deux emploie $60 \%, 70 \%$ et même 90 \% d'immigrants. De plus, la moitié des entreprises (54\%) sont homogènes sur le plan ethnique, c'est-à-dire que les dirigeants, les superviseurs et les travailleurs sont issus des mêmes pays d'origine par exemple l'Inde, le Congo, le Liban et autres. On compte également $25 \%$ d'entreprises présentant une grande mixité ethnique entre les dirigeants, les superviseurs (canadiens) et les employés par exemple d'origine haïtienne, coréenne, marocaine ou autres. Contrairement à ce que nous présumions au départ, les travailleurs immigrants ne sont pas récemment arrivés: la moitié sont au Canada depuis plus de 10 ans et $59 \%$ ont plus de 5 ans l'ancienneté dans l'entreprise.

La majorité ( $82 \%)$ des entreprises de notre échantillon ont plus de 21 travailleurs. Certaines entreprises saisonnières ont plus de 50 travailleurs, dont la taille de la maind'œuvre fluctue entre 12 et 75 travailleurs selon les cycles de production. Seulement $18 \%$ (5/28) des entreprises ont entre 10 et 20 travailleurs.

\subsection{Initiation à la tâche : élément d'une culture de SST}

Interrogées sur les pratiques de formation qu'elles ont établies, 92,9\% (26/28) des entreprises disent initier leurs nouveaux employés à leurs tâches. Parmi les PE de notre échantillon qui initient les nouveaux employés, la majorité (15/26) y consacrent plus d'une journée. Certaines (5/28) ont une pratique supervisée qui peut durer quelques mois (mentorat, apprentis et compagnonnage). Plus de la moitié des entreprises, 64,3\% 
(18/28), donnent une orientation structurée en santé et sécurité au travail à l'ensemble de leurs nouveaux employés lors de l'embauche. Cette orientation aborde principalement les méthodes de travail sécuritaires et les équipements de protection individuelle.

L'intensité de ces initiations varie beaucoup. Deux entreprises ( $n^{\circ} 2$ et $n^{\circ}{ }^{5}$ ) n'ont pas d'activité pour initier leurs nouveaux employés lors de l'embauche. Pourtant, ces deux entreprises sont dans des groupes prioritaires de la fabrication de produits en métal et des revêtements métalliques. Caractéristique importante, ces deux entreprises font partie des entreprises comptant $50 \%$ et plus de travailleurs immigrants. Dans les deux cas, les entreprises ont des comités de SST plus ou moins fonctionnels, les rencontres y sont plus ou moins formelles et régulières. Ces comités ne sont pas paritaires. Ils ont été créés il y a moins de trois ans alors que les entreprises font des affaires depuis plus de 30 ans.

Dans la première entreprise ( $\left.n^{\circ} 2\right)$, non syndiquée, $90 \%$ de la main-d'œuvre est immigrante originaire de cinq pays dont $70 \%$ viennent des Indes. Parmi ceux-ci, plus de la moitié ont immigré (60\%) depuis moins de cinq ans. On y compte plusieurs langues de travail dont les deux principales sont l'anglais et l'hindi, les deux langues parlées par les superviseurs et le dirigeant de l'entreprise. Bien qu'elle n'ait pas d'activités de formation structurées, l'entreprise aura recours, selon les besoins, à un ancien employé pour former ses compatriotes aux mesures de SST en hindi. Malgré que l'entreprise ne reconnaisse pas ces activités comme une formation structurée, elle est l'une des deux entreprises qui ont adapté l'enseignement sur les normes minimales (secourisme et chariot élévateur) et le port des équipements personnels individuels dans la langue maternelle de ses employés.

Dans la seconde entreprise ( $n^{\circ}$ 5), également non syndiquée, $75 \%$ de la main-d'œuvre est immigrante. Parmi ce nombre, $20 \%$ ont immigré depuis moins de cinq ans. Les travailleurs sont issus d'au moins six communautés autres que canadiennes. Les langues de travail sont principalement l'anglais, le français, le portugais et l'arménien. Dans cette deuxième entreprise, il y a également une certaine homogénéité culturelle et linguistique entre les superviseurs, les employés et les propriétaires-dirigeants. Contrairement à l'entreprise précédente, les besoins d'initiation à la tâche se font en français ou en anglais.

33 Au contraire des entreprises $n^{\circ} 2$ et $n^{\circ} 5$, deux entreprises se distinguent : la première $\left(n^{\circ}\right.$ 52) prévoit entre deux semaines et trois mois de compagnonnage selon les besoins $d u$ poste, et la seconde ( $\left.\mathrm{n}^{\circ} 106\right)$, un an de compagnonnage à tous les employés.

Dans l'entreprise $\mathrm{n}^{\circ}$ 52, non syndiquée, $91 \%$ de la main-d'œuvre est immigrante. Les travailleurs sont issus d'au moins quatre communautés autres que canadiennes. Les langues de travail sont principalement l'anglais, le français, l'arabe et l'espagnol. Dans cette entreprise, il y a également une certaine homogénéité culturelle et linguistique entre les superviseurs, les employés et le propriétaire-dirigeant originaires du MoyenOrient. Pour certains nouveaux employés, le compagnonnage se fait en arabe.

Dans l'entreprise nº 106, entreprise syndiquée, tous les travailleurs sont canadiens dont la très grande majorité provient du Québec. L'entreprise très stable a embauché peu de nouveaux travailleurs au cours des cinq dernières années. Les langues de travail sont le français et l'anglais. 


\subsection{Formation : un levier culturel de SST important}

On note que $92,9 \%(26 / 28)$ des entreprises donnent une formation aux normes minimales à leurs nouveaux employés concernant les chariots élévateurs, le secourisme, les ponts roulants, le cadenassage, le système d'information sur les matières dangereuses utilisées au travail (SIMDUT) et les nacelles. Certaines formations ne sont données qu'aux détenteurs de postes spécifiques. Par contre, plus de la moitié $64,3 \%(18 / 28)$ assurent que le quart de leur personnel a, en cours d'emploi, des formations aux normes minimales. Ces formations comme celles sur le bruit, le SIMDUT ou le monoxyde de carbone sont considérées onéreuses en matière de temps, puisqu'elles exigent que les travailleurs soient libérés entre 8 et 16 heures, selon le type de formation ciblée.

Une entreprise $\left(n^{\circ} 18\right)$ a toutefois fait preuve d'ingéniosité pour mettre à jour les formations en SST pour tous ses employés. Profitant d'une période de ralentissement, la directrice des ressources humaines a fait une demande de subvention (89000\$) à un organisme régional dont la mission est la formation et la mise à jour des compétences de la main-d'œuvre ${ }^{4}$. Cet organisme dispose de programmes fort intéressants. Ainsi, la directrice a pu obtenir pour une période de six mois des fonds lui permettant d'engager les formateurs, de payer les formations externes et d'obtenir des compensations salariales d'une à deux journées par semaine, et ce, pour quelques semaines, selon les besoins des travailleurs. Soulignons que cette directrice des ressources humaines est détentrice d'un baccalauréat en administration et d'un certificat en SST. Elle connaît les programmes de soutien aux entreprises et les conditions d'éligibilité.

Une autre entreprise $\left(n^{\mathrm{Q}} 3\right)$ a fait de la formation en secourisme, une compétence ajoutée au métier, une compétence reconnue bien qu'elle ne soit pas dans un secteur hautement à risque. Devant l'engouement de ses travailleurs pour ces formations en secourisme, elle a attribué cette formation en procédant au tirage au sort de façon cyclique. Cette entreprise compte également un directeur des $\mathrm{RH}$ détenteur d'une technique en ressources humaines.

les entreprises de l'échantillon, certaines sont des filiales d'entreprises canadiennes ou internationales (10/28). Celles-ci disposent généralement d'un cadre de référence en matière de SST beaucoup plus directif. L'initiation à la tâche est généralement obligatoire avec des mesures de compagnonnage très formelles. Par exemple, dans une fonderie ( $\mathrm{n}^{\circ}$ 103), l'initiation aux postes et la formation en SST sont incontournables et conditionnelles à l'obtention des cartes de compétence. Cette entreprise est régie à la fois par les normes nationales du secteur de production des métaux, les normes internationales du consortium auquel elle appartient, et également les normes du syndicat national des fondeurs. Parmi les compétences évaluées par le syndicat pour obtenir des cartes, il y a l'obligation de conformité aux méthodes sécuritaires de travail et aux mesures d'urgence dont celle d'apporter assistance à ses collègues en cas d'accident. Cette entreprise n'emploie aucun travailleur immigrant.

De façon générale, on note que les entreprises non syndiquées embauchant des travailleurs saisonniers et immigrants ne bénéficient pas d'encadrement aussi formel que l'ensemble des travailleurs. La vérification de la conformité des consignes de sécurité est quasi inexistante dans ces entreprises où les tâches sont considérées moins spécifiques. Néanmoins, dans une entreprise, une confiserie ( $n^{\circ} 36$ ), la directrice cumulant les fonctions de chef de la production et des ressources humaines constate, lors du retour des 
travailleurs saisonniers, que tous ses nouveaux employés, majoritairement hispanophones, n'ont rien saisi des consignes de SST enseignées lors de leur initiation à la tâche. Parce qu'elle s'exprime aisément en espagnol, elle a pu observer cette lacune. Devant l'échec total de cette initiation par un formateur externe, l'entreprise décide d'adapter ses formations. Elle choisit un de ses employés hispanophones parmi les plus anciens et le désigne comme formateur. Elle lui confie le mandat d'initier les nouveaux employés et d'assurer les mises à jour des formations en SST, et ce, pour tout le personnel qui est à $80 \%$ immigrant dont $60 \%$ hispanophone. Cette initiative a nécessité des investissements pour former à l'interne le travailleur et adapter le matériel; des investissements qui ont été assumés entièrement par l'entreprise.

\subsection{Autres acteurs contribuant aux rituels et à une culture de la SST}

41 Pour les formations en cours d'emploi et les mises à jour des équipements et procédés sécuritaires de travail, les PE font généralement appel à des formateurs externes. Les associations sectorielles paritaires (ASP) sont une source de référence pour certaines d'entre elles, 10/28 en font partie. Par contre, deux entreprises ( $\mathrm{n}^{\text {os }} 46$ et 101) déplorent le fait d'être classées dans une ASP qui ne correspond pas à leur secteur de production. Ces dernières considèrent l'expertise à laquelle elles ont accès inappropriée à leurs besoins. Si certaines ne connaissent pas leur ASP, d'autres font partie d'une mutuelle de prévention (14/28) dont elles apprécient les compétences.

42 Une entreprise ( $\left.n^{\circ} 20\right)$ spécialisée dans la fabrication et le nettoyage de moules de métal pour la boulangerie a eu recours aux conseils de son ASP pour redresser toute sa structure de SST. Celle-ci a accompagné l'entreprise dans ses démarches pour constituer un comité de SST, en définir le mandat, et pour départager les revendications syndicales et les besoins de SST dont les besoins de formation. Cette entreprise est composée à $86 \%$ d'immigrants, tous immigrés depuis plus de 10 ans. Les travailleurs et les superviseurs originaires d'Amérique centrale et du Sud, d'Afrique, des Caraïbes et d'Europe de l'Est utilisent trois langues au travail : le français, l'anglais et l'espagnol.

43 Les thèmes de formation les plus communs sont les équipements personnels individuels (ÉPI), les méthodes et les outils de travail sécuritaires. Les autres thèmes touchent les machines et les outils, les risques à la santé dont le bruit et les solvants. Les affiches et les dépliants servent à informer le personnel de certaines nouveautés en SST.

Les professionnels de la santé au travail des CSSS sont souvent mis à contribution par les PE pour donner plus de poids et de crédibilité afin d'introduire de nouvelles pratiques. Les répondants de SST dans les PE et les professionnels de SST des CSSS reconnaissent qu'aucune formation et aucun matériel n'est adapté ou traduit pour les travailleurs issus de l'immigration. La majorité présume que leur maîtrise des langues officielles lors de l'embauche est suffisante pour saisir et intégrer les consignes de SST.

\subsection{Légendes urbaines de la SST}

45 Outre cette présomption d'aisance à comprendre les consignes de SST dans une langue autre que leur langue maternelle, c'est tout le régime qui est mal compris. Deux entreprises ont souligné les incompréhensions des travailleurs immigrants quant au régime de santé et de sécurité au travail, incompréhensions qui mènent à des distorsions pouvant être apparentées à des abus. 

la direction des ressources humaines (DRH), lors d'une période de licenciement, du " chômage médical ». Ayant entendu parler que le « chômage médical était plus avantageux que le chômage régulier, les travailleurs récemment immigrés voulaient savoir comment choisir leur chômage ». La DRH a rapidement fait une mise au point leur expliquant la différence entre ces deux régimes, la CSST et l'assurance-emploi, et leurs impacts financiers sur l'entreprise.

Une autre entreprise $\left(\mathrm{n}^{\circ} 46\right)$ a connu également une période de légendes urbaines. Cette fois les «travailleurs immigrants croyaient que s'ils se blessaient, le programme de réadaptation leur permettrait d'être réhabilités dans leur profession ou leur métier acquis dans leur pays d'origine. L'indemnisation était perçue comme une avenue pour obtenir une reconnaissance de leurs compétences antérieures ». Le DRH de cette entreprise a jugé opportun d'en saisir un conseiller à la CSST. Ce dernier est venu expliquer aux travailleurs immigrants les fondements du régime. $\mathrm{Si}$, dans le passé, certains travailleurs étrangers blessés ont obtenu des assignations à d'autres postes ou fonctions temporaires qui tenaient compte de leurs compétences antérieures à l'emploi occupé lors de la lésion, ces cas sont exceptionnels.

\subsection{Culture de prévention chez les gestionnaires}

La formation des gestionnaires influence la qualité du management de la SST dans les entreprises. Parmi les dix entreprises ayant des répondants de SST bénéficiant soit d'une formation spécifique en SST (certificat ou diplôme d'études de $2^{\mathrm{e}}$ cycle) ou d'une formation en gestion des ressources humaines ou en relations industrielles (baccalauréat ou master), la majorité (8/10) gère les mesures de SST de façon satisfaisante selon les professionnels de SST des CSSS partenaires. Ces derniers jugent les compétences et les habiletés des répondants de SST dans les PE selon un certain nombre de critères plus ou moins explicites dans leur grille d'évaluation des besoins des entreprises utilisée lors des visites. Les compétences des répondants de SST sont mesurées selon leurs capacités à déceler et évaluer les risques de SST, à transmettre aux dirigeants les besoins des travailleurs en matière de SST, à respecter la réglementation, à lancer des projets, et finalement à implanter des programmes ou des mesures de SST. Les professionnels de SST des CSSS jugent les entreprises selon leurs capacités à implanter des programmes ou à appliquer des recommandations de façon complète, partielle (une seule partie des actions), négligente (entraînant de nouveaux risques) ou en délaissant le programme ou les mesures en cours d'implantation.

Les critères des professionnels de SST pour juger des compétences des entreprises et des responsables de SST ne font aucunement référence aux formations acquises par ces derniers. Un constat également noté dans nos résultats alors que les répondants de SST très scolarisés, mais ne possédant aucune formation en SST évaluent très sévèrement leurs connaissances en cette matière. Un chef de production, détenteur d'un Ph.D. en chimie ( $\left.n^{\circ} 5\right)$, formé en Europe de l'Est, constate avec beaucoup d'humilité que la culture de la SST est fort différente en Amérique du Nord, elle est beaucoup plus complexe et structurée en ce qui concerne les lois, les normes, les pratiques et la surveillance.

«Malheureusement, c'est à coup d'amendes et de mesures coercitives qu'un dirigeant formé à l'étranger sera saisi de cette culture et de sa complexité. Il n'y a pas d'indication ni de lieux où il est possible de s'initier en tant que dirigeant. » 
de la SST. C'est le cas de certaines entreprises, où les modes de production n'ont pas évolué au même rythme que la mise à jour des normes de SST. Leurs dirigeants peinent à s'ajuster à cette culture de la SST considérée exigeante et contraignante ( $\left.n^{\circ} 47\right)$.

51 Une entreprise (no 103) se distingue par son système exemplaire de compagnonnage et de monitorage des réflexes quotidiens de prévention. Le monitorage des réflexes consiste à relever quotidiennement les incidents ou les méthodes de travail qui sont potentiellement à risques. Chacun des quatre dirigeants doit à tour de rôle, au cours d'une semaine, se rendre dans les unités de production et procéder à des vérifications des méthodes de travail, du port des équipements de protection, des tests thermiques, de toxicité et de bruit. Toutes les vérifications sont consignées dans un registre informatisé partagé entre la direction et les employés pour signifier tous les écarts de pratique. Par ce moyen, l'entreprise crée une cohérence entre la culture de la SST et les pratiques de gestion des ressources humaines (GRH). Cette entreprise est l'une des trois entreprises de notre échantillon qui n'embauchent aucun immigrant. Il y a une complète homogénéité de la main-d'œuvre: homme, francophone, canadien de naissance, âgé de 25 à 55 ans. Soulignons que dans cette entreprise, le responsable de la SST est détenteur d'un certificat universitaire de $1^{\text {er }}$ cycle en SST, il occupe un poste à temps plein de coordination de la SST et bénéficie d'un cadre de la prévention dicté par le siège social de l'entreprise, une multinationale.

\section{Discussion}

52 Nos résultats, comme dans d'autres études (Nash, 1996; Chatigny et al., 2006; Vézina et al., 2000), font ressortir l'importance de l'initiation des travailleurs à leurs tâches, de la formation et de l'information en cours d'emploi, de leur degré de participation dans la prévention en SST pour créer une culture de prévention de la SST. Cela est d'autant plus important que cette dernière se fonde sur des bases plus larges que la seule rationalité instrumentale de la gestion du risque et des processus. Cela suppose que des acteurs internes soient clairement désignés comme les porteurs de cette culture, qu'ils soient à la fois maîtres des savoir-faire (compétences, certifications et expériences en SST) pour instaurer sur une base cyclique des occasions de revoir, reprendre et bonifier les pratiques de SST. Il nous apparaît important dans un contexte pluriethnique et plurilinguistique d'introduire ces pratiques sous l'angle de la culture et du rituel, prenant en compte les perceptions, les différences de représentations et les attitudes des acteurs pour apporter des changements de pratiques et de comportements dans toutes les sphères de la prévention (traumatismes routiers, tabagisme, alcoolisme, obésité, etc.).

Il semble trop facile d'incriminer les conditions structurelles et la précarité des moyens des PE pour expliquer l'inertie dans la prise en charge des mesures de SST. Nos résultats indiquent qu'au-delà des conditions structurelles et matérielles, il est possible de créer une culture de SST si les dirigeants accordent une importance symbolique à la santé comme valeur "sacrée ». Certaines entreprises réussissent à sacraliser la santé en s'appuyant sur un idéal de métier marqué par des règles exigeantes (ex. : certification), des modes d'inclusion dans un collectif (ex.: syndicat) et légitimé d'activités de prévention (ex. : carte de compétences). Pour ce faire, les moyens et les méthodes pour éviter les dangers s'articulent dans une logique cohérente. En commençant par

Perspectives interdisciplinaires sur le travail et la santé, 13-2 | 2011 
l'initiation aux tâches à l'embauche, pour ensuite se consolider en cours d'apprentissage par les pratiques de supervision ou de compagnonnage et devenir une référence au cours des années par diverses activités mobilisatrices de surveillance et d'analyse des pratiques de SST. Certains acteurs internes sont alors appelés à devenir des maîtres qui mobilisent et orientent les travailleurs, non pas sous la contrainte de la conformité des actions aux règlements et aux lois de SST, mais bien dans un rituel partagé qui unifie les travailleurs autour des meilleures pratiques. Nos résultats indiquent que les dirigeants ayant acquis une formation en SST, soit un certificat de SST ou quelques cours dans le cadre d'une formation en gestion acquise au Canada, s'acquittent beaucoup plus facilement de cette tâche que les autres, et ce, même si ces derniers sont très scolarisés.

Les légendes urbaines au sujet du régime de SST illustrent bien que les PE embauchant des travailleurs immigrants doivent redoubler d'efforts pour transmettre cette culture. Cette transmission sera d'autant plus hasardeuse si les propriétaires-dirigeants euxmêmes issus de l'immigration sont originaires de pays où la culture de SST est inexistante.

La composition ethnique des entreprises de notre échantillon fait état d'une très grande diversité des origines de la main-d'œuvre au sein des PE. C'est peut-être ici un élément important pour comprendre ces dynamiques plus ou moins fonctionnelles pour l'établissement d'une culture de SST dans les PE. Bien que cette étude ne dispose pas d'observations ethnographiques des pratiques de SST au sein des entreprises, au sens traditionnel de l'ethnographie (observations participantes par des personnes intégrées dans le quotidien du groupe observé), il est possible d'avancer quelques éléments explicatifs. Les entreprises mixtes sur le plan de la diversité ethnique, à savoir des dirigeants et des superviseurs d'origine canadienne avec des employés issus de l'immigration en provenance de divers pays, semblent redoubler d'effort pour créer un environnement propice à une culture de SST. Par contre, les entreprises homogènes sur le plan ethnique, où les dirigeants, les superviseurs et les employés sont immigrants des mêmes pays, partagent une culture de SST qui s'apparente davantage à celle de leur pays d'origine où les lois et les règlements de SST n'ont pas souvent d'équivalence avec ceux du Canada.

Une approche culturelle et ritualisée de la prévention en SST oblige à prendre en compte d'autres considérations liées au processus et au statut d'immigrant, aux influences des expériences culturelles d'origine dans les pays de provenance des travailleurs et des directions, et de leur situation dans le pays actuel. Ces dimensions interviennent fortement dans la vision et l'importance accordée à la santé, au risque, à la santé au travail, aux rapports à l'autorité, au collectif de travail, à la participation, au sens et à l'importance du travail lui-même et au métier. Par exemple, il est fréquent de trouver dans ces PE des travailleurs immigrants surqualifiés pour les postes qu'ils occupent, ce travail étant alors uniquement un gagne-pain pour assurer la survie de la famille. Toujours en attente de trouver un meilleur emploi correspondant à leurs compétences initiales, les travailleurs immigrants s'investissent peu ou pas dans ces emplois transitoires.

Pour les chefs d'entreprise eux-mêmes immigrés du Liban, de la Roumanie, d'Haïti, etc., les impératifs de la culture de la SST à la québécoise risquent fort de constituer un univers parallèle, peu intégré à la production. De plus, ils ont appris leur métier où le recrutement de la main-d'œuvre était facile, le taux de chômage était élevé, les préoccupations pour préserver une main-d'œuvre ne faisaient pas partie des enjeux de 
gestion. Sans y être opposés, ces dirigeants eux-mêmes issus de l'immigration ne savent pas comment intégrer la culture de SST à la production.

Cette étude exploratoire présente des limites méthodologiques. L'échantillon est constitué d'entreprises volontaires, ayant bénéficié de l'intervention d'une équipe de la CSST, et démontrant leur bonne volonté. Ces entreprises n'ont pas été tirées au hasard, elles font partie des secteurs de production ciblés dans les plans de surveillance de la CSST. Bref, plusieurs considérations méthodologiques pourraient bonifier une telle étude : augmenter l'échantillon, ajouter certains secteurs de production non prioritaires, et solliciter des entreprises par l'intermédiaire d'un regroupement ou d'une fédération de PE.

Comme nous l'avons souligné à quelques reprises, une démarche de type ethnographique fondée sur des observations directes de pratiques de formation ou d'initiation à l'embauche permettrait d'étayer plus avant notre piste de recherche sur l'importance de pratiques ritualisées dans l'établissement d'une culture SST dans les PE embauchant des travailleurs immigrants. Il conviendrait aussi d'examiner de plus près, par une étude longitudinale, les trajectoires de carrières des travailleurs immigrants et d'y associer une analyse des représentations de la notion de santé, de bien-être et de risque au travail. Finalement, il serait important d'explorer l'hypothèse que les travailleurs immigrants s'investissement moins, participent moins aux mesures de SST parce que les emplois qu'ils occupent dans les PE étaient au départ des emplois de transition.

\section{Conclusion}

L'analyse des dynamiques de prise en charge des PE a été minutieusement étudiée sous l'angle des structures et des moyens dont disposent les acteurs pour ériger une culture de SST au sein des PE. Il devient impératif en ce $21^{\mathrm{e}}$ siècle, alors que l'embauche des travailleurs immigrants est une solution à la pénurie de main-d'œuvre, que ces dynamiques de prise en charge de la SST tiennent compte de la composition ethnique des travailleurs, de leur parcours d'immigration et d'insertion sur le marché du travail et de leur conception de la santé au travail. Les travailleurs immigrants se sentent-ils concernés par la SST des emplois qu'ils n'occupent que temporairement, des emplois qui ne correspondent pas à leurs compétences initiales? Même s'ils sont dans une contradiction d'insertion professionnelle, il n'en demeure pas moins que s'ils se blessent ou deviennent handicapés, ils seront limités dans l'exercice de leur emploi actuel et à l'égard de tous les autres emplois qu'ils souhaiteraient occuper.

61 Au-delà de l'adaptation linguistique des mesures de SST, des formations et des informations techniques de base, des activités appropriées pour instaurer une forte culture de santé et sécurité au travail constituent l'essentiel de la prévention et reposent sur une action ritualisée, répétée et centrée sur le caractère "sacré » de la santé de tous les travailleurs. Notre recherche montre qu'un apport théorique et des exemples de rites, de rituels, les symboles du risque et de la sécurité au travail seraient des plus utiles et pertinents pour développer et guider les éventuels travaux menés au sein des entreprises qui embauchent un nombre important de travailleurs immigrants.

Ce projet de recherche a obtenu le soutien financier de l'Institut de recherche Robert-Sauvé en santé et en sécurité du travail pour sa réalisation (IRSST) (099-682). 


\section{BIBLIOGRAPHIE}

G. Baril-Gingras, M. Bellemare, J. P. Brun. Interventions externes en santé et en sécurité du travail : influence du contexte de l'établissement sur l'implantation de mesures préventives. Relations industrielles, 2006, 61 (1) : 9-43.

M. P Brown,. et al. 2002. California's Immigrant Workers Speak Up About Health and Safety in the Workplace.. Health \& Safety policy brief. Labor occupational safety and health program (LOSH). 4 p.

M. R. Charpentier-Roy, M. Simard, A. Marchand, F. Ouellet. « Pour un modèle renouvelé d'intervention en santé au travail dans les petites entreprises ». Relations industrielles/Industrial Relations, 2001; 56 (1) : 165-194.

C. Chatigny, A. Balleux, M. Martin, J. Grenier, S. Ouellet, M. Corbeil, M. Laberge, D. Rochette. Étude exploratoire des dynamiques de formation et d'apprentissage : apprentissage des tâches et prévention des troubles musculo-squelettiques dans trois entreprises du secteur avicole. Études et recherches / Rapport R-464, Montréal, IRSST, 2006, 80 pages.

E. Cloutier. Enjeux liés à la problématique du vieillissement dans les milieux de travail, Colloque 2009 IRSST, 27 octobre 2009, Le vieillissement de la main-d'œuvre et la SST, Recueil des présentations. 2009.

L. Desmarais, M. Foucault. Mesure de performance pour les comités de SST. Le Grand Rendezvous santé et sécurité du travail, Palais des Congrès, Montréal. 17 novembre 2009.

J. Eakin. Leaving It Up to the Workers: Sociological Perspectives on the Management of Health and Safety in Small Workplaces, International Journal of Health Services, 1992; 22 (4): 689-704.

J. Eakin, E. MacEachen. Health and the social relations of work: a study of the health-related experiences of employees in small workplaces. Sociology of Health and Illness, 1998; 20 (6): 896-914.

J. Eakin, F. Lamm, J. J. Limborg. International Perspectives on the Promotion of Health and Safety in Small Workplaces. In Frick K., Jensen P.L., Quinlan M., Wilthagen T., (ed.). Systematic Occupational Health and Safety Management, Perspectives on an International Development, Amsterdam, Pergamon; 2000: 227-247.

D. E. Eggerth. Occupational Safety Risk Perceptions and Acceptance among Hispanic Immigrants in the United States. 12th International Metropolis Conference, 8 - 12 October, 2007; Melbourne, Australia.

M. Fellous. À la recherche de nouveaux rites : rites de passage et modernité avancée. L'Harmattan, Logiques sociales, France. 2001. 245 pages.

M. Flynn. Occupational Health Disparities among Hispanic Immigrants in the United States. National Institute for Occupational Safety and Health. Centers for Disease Control and Prevention. 12th International Metropolis Conference, 8 - 12 October, 2007. Melbourne, Australia.

S. Gravel. Analyse du parcours d'indemnisation de travailleurs immigrants victimes de lésions professionnelles. Thèse de doctorat, Faculté des études supérieures de l'Université de Montréal, pour l'obtention d'un Ph.D. en santé publique. 2006. 
N. Lashay, B. J. Burgel, R. Harrisson, L. Israel, J. Chan, C. Cisic, J. C. Pun, K. Fong, Y. Shin. We Spend Our Days Working in Pain: A Report on Workplace Injuries in the Garment Industry, Asian Immigrant Women Advocates, 2002.

G. Legendre 2011. Les comités de santé et de sécurité au travail dans les petites entreprises montréalaises qui embauchent des travailleurs immigrants. Mémoire de maîtrise en administration des affaires, profil gestion des ressources humaines. Université du Québec à Montréal; 133 pages.

M. Nash. Language barriers and safety. Speaking in tongues. Occupational Health and Safety Canada, 1996, 6 (5), septembre - octobre.

J. J. Paques, R. Bourbonnière, R. Daigle, P. Doucet, P. Masson, P. Michaud, J. Lane, J. Tardif. Transfert de compétences en formation sur la gestion de la sécurité des machines et les moyens de protection. Études et recherches / Rapport R-394, Montréal, IRSST, 2005, 105 pages.

T. T. Paterson. Les rites émotifs dans les organisations industrielles. In Huxley Julian. 1991. Le comportement rituel chez l'homme et l'animal. 1971, p. 291-299.

M. Quinlan. Workers' compensation and the challenges posed by changing patterns of work: evidence from Australia. Policy and Practice in Health and Safety, 2004; 2(1): 25-52.

M. Quinlan, C. Mayhew. Precarious employment and workers' compensation. International Journal of Law and Psychiatry, 1999; 22 (5 et 6): 491-520.

J. C. Robinson. Trends in racial inequity and exposure to work-related hazards, 1968-1986. Milbank Q. 1987; 65 (suppl.): 404-420.

M. Segalen. Rites et rituels contemporains. Armand Colin, Paris. 2005. 128 pages.

M. Simard, A. Marchand. La participation des travailleurs à la prévention des accidents du travail : formes, efficacité et déterminants. Études et recherches / Rapport R-154, Montréal, IRSST, 1997; 40 pages.

P. Smith. Institute for Work \& Health, Toronto, Canada. Examining the risk of work-related injuries and compensation after injury among Canadian immigrants. 12th International Metropolis Conference, 8 - 12 October, 2007. Melbourne, Australia.

F. Soto Mas, R. L. Papenfuss, J. Guerrero. Hispanics and worksite health promotion: review of the past, demands for the future. J Community Health, 1997; 22 (5): 361-371.

K. C. Stange, D. Strogatz, V. J. Schoenbach, C. Shy, B. Dalton, A. W. Cross. Demographic and Health Characteristics of Participants and Nonparticipants in a Work Site Health-Promotion Program.J Occup Med, 1991; 33 (4): 474-478.

Statistique Canada. 2008. Établissements employeurs selon la taille de l'établissement dans les provinces et territoires, décembre 2007. Registre des entreprises, Internet.

J. Sum. Navigating the California Workers' Compensation System: The injured Worker's Experience. San Francisco: California Commission on Health and Safety and Workers' Compensation, 1996.

J. Taylor. Labour Market and Fiscal Impacts of Immigration. International Migration, Papers, no. 10. Geneva: ILO, 1996.

V. Turner. Le Phénomène rituel. Structure et contre structure, PUF. 1990.

A. Van Gennep. Le folklore. Paris, Librairie Stock. 1924. 124 pages. 
N. Vézina, J. Prévost, A. P. Lajoie. Élaboration d'une formation à l'affilage des couteaux dans six usines d'abattage et de transformation du porc : une étude ergonomique. Études et recherches / Rapport R-243, Montréal, IRSST, 2000, 55 pages.

I. Vickers, R. Baldock, D. Smallbone, J. Phillips, I. Ekanem. Cultural influences on health and safety attitudes and behaviour in small businesses. Contract Research Report No. RR150: HSE. www.hse.gov..uk/research/rrpdf/rr150.pdf. 2003.

D. Walters. Employee representation on health and safety in small enterprises; a trade union initiative in agriculture. Employee Relations, 1992; 20 (2): 164-179.

D. Walters. The Identification and Assessment of Occupational Health and Safety Strategy in Europe. Volume 1: The national situations. European Foundation for the Improve of Living and Working Conditions. Dublin, Ireland, 1996.

D. Walters. Health and Safety in Small Enterprises: European Strategies for Managing Improvement, New York, P.I.E. - Peter Lang, 2001, 404 p.

D. Walters. Workers representation and health and safety in small enterprises in Europe. Industrial Relations Journal, 2004; 35 (2): 169-186.

D. Walters. Within Reach? Managing Chemical Risks in Small Enterprises. Work Health and Environment Series. Raywood Publishing Company Inc. Amityville, New York, 2008.

A. Williams, A. Mason, J. Wold. Cultural Sensitivity and Day Care Workers: examination of a worksite based cardiovascular disease prevention project. AAOHN. 2001.

J. Windau. Occupational Fatalities among the Immigrant Population. Compensation and Working Conditions, 1997; Spring: 40-44.

\section{NOTES}

1. Au Québec, $95 \%$ des employeurs sont des PE (4).

2. La trajectoire migratoire inclut les pays hôtes avant l'établissement (pays de transition et les camps de refuge) et les conditions d'établissement (programme d'admission, durée de séjour dans le pays hôte) (Gravel S., Battaglini A. 2000. Culture, santé et ethnicité: vers une santé publique pluraliste. Direction de la santé publique de Montréal-Centre. 241 pages.

3. Culture: ensemble des us et coutumes, des manifestations religieuses, artistiques, intellectuelles et politiques appuyées par des institutions qui définissent et distinguent un groupe ou une société.

4. Programme SERRÉ, offert par le Conseil régional de Montréal des partenaires pour la maind'œuvre, conseil rattaché à la structure consultative du ministère de l'Emploi et de la Solidarité sociale.

\section{RÉSUMÉS}

Le présent article traite une partie des résultats d'une étude portant sur les stratégies favorables à la prise en charge des mesures de santé et sécurité au travail (SST) dans les petites entreprises 
montréalaises (PE) embauchant une main-d'œuvre immigrante. Dans cet article est abordée entre autres l'influence de l'immigration sur la capacité et la motivation des superviseurs et des travailleurs à participer à la gestion des mesures de SST dont celles de la formation et de l'initiation à la tâche.. L'échantillon comprend $28 \mathrm{PE}$ de Montréal ayant entre 10 et 50 travailleurs répartis en deux groupes : a) PE d'observation ayant $25 \%$ et plus de travailleurs immigrants $(n=19)$; b) PE de comparaison ayant $75 \%$ et plus de travailleurs nés au Canada ( $n=9)$. Les résultats indiquent que les compétences des dirigeants et la formation acquise dans leur pays d'origine orientent l'adoption de rituels de prévention dès l'embauche des nouveaux travailleurs. Enfin, malgré l'importance de la présence de travailleurs immigrants dans les PE, seulement deux entreprises de notre échantillon ont adapté leur formation aux nouveaux employés immigrants.

This study examines the influence of immigration on motivation and ability of supervisors and workers in small business (SB) to participate in health and safety measures (OSH) management. The sample consists of 28 Montreal's SB between 10 and 50 workers divided into two groups: a) SB observation with $25 \%$ or more immigrant workers ( $n=19)$; b) SB compared with $75 \%$ and more Canadian-born workers $(n=9)$. The results indicate that managers' competencies and training developed in their country of origin condition their approach concerning hiring and training rituals. Despite the importance of immigrant workers' presence, only two companies in our sample have adapted their training for new immigrant employees.

El presente artículo trata una parte de los resultados de un estudio sobre las estrategias favorables a la aplicación de las medidas de salud y seguridad en el trabajo (SST) en las pequeñas empresas de Montreal (PE) que contratan una mano de obra inmigrante. En este articulo se abordan entre otras cosas : la influencia de la inmigración sobre la capacidad y la motivación de los supervisores y de los trabajadores a participar en la gestión de las medidas de SST, entre ellas la formación e iniciación a la tarea. La muestra comprende 28 PE de Montreal que contratan de 10 a 50 trabajadores repartidos en dos grupos : a) PE de observación, que cuentan con $25 \%$ y más trabajadores inmigrantes ( $n=19):$ b) PE de comparación, con 75\% y más trabajadores nacidos en Canadá ( $n=9$ ). Los resultados indican que las capacidades de los dirigentes y la formación adquirida en sus países de origen orientan la adopción de rituales de prevención desde la contratación de los nuevos trabajadores. Por ultimo, a pesar de la importancia de la presencia de trabajadores inmigrantes en las $\mathrm{PE}$, solamente dos empresas de nuestra muestra adoptaron su formación a los nuevos empleados inmigrantes.

\section{INDEX}

Mots-clés : formation, initiation à la tâche, travailleurs immigrants, petites entreprises, adaptation culturelle et linguistique

Keywords : training, introduction to the task, immigrant workers, small businesses, cultural and linguistic adaptation

Palabras claves : formación, iniciación a la tarea, trabajadores inmigrantes, pequeñas empresas, adaptación cultural y lingüística

\section{AUTEURS}

\section{SYLVIE GRAVEL}

gravel.s@uqam.ca, Département d'organisation et ressources humaines, École des sciences de la gestion, UQAM, Case postale 8888, succursale Centre-ville, Montréal (Québec) H3C 3P8 


\section{JACQUES RHÉAUME}

rheaume.jacques@uqam.ca, Département de communication sociale et publique, Université du Québec à Montréal, Case postale 8888, succursale Centre-ville, Montréal (Québec) H3C 3P8

\section{GABRIELLE LEGENDRE}

legendre.gabrielle@uqam.ca, Département d'organisation et ressources humaines, École des sciences de la gestion, UQAM, Case postale 8888, succursale Centre-ville, Montréal (Québec) H3C $3 \mathrm{P} 8$ 